\title{
RTDF2007-46009
}

\section{EQUATIONS OF MOTION FOR TRAIN DERAILMENT DYNAMICS}

\author{
D.Y. Jeong \\ M.L. Lyons \\ O. Orringer \\ A.B. Perlman \\ U.S. Department of Transportation \\ Volpe National Transportation Systems Center \\ Cambridge, Massachusetts, USA
}

\begin{abstract}
This paper describes a planar or two-dimensional model to examine the gross motions of rail cars in a generalized train derailment. Three coupled, second-order differential equations are derived from Newton's Laws to calculate rigid-body car motions with time. Car motions are defined with respect to a right-handed and fixed (i.e., non-rotating) reference frame. The rail cars are translating and rotating but not deforming. Moreover, the differential equations are considered as stiff, requiring relatively small time steps in the numerical solution, which is carried out using a FORTRAN computer code. Sensitivity studies are conducted using the purpose-built model to examine the relative effect of different factors on the derailment outcome. These factors include the number of cars in the train makeup, car mass, initial translational and rotational velocities, and coefficients of friction. Derailment outcomes include the number of derailed cars, maximum closing velocities (i.e., relative velocities between impacting cars), and peak coupler forces. Results from the purpose-built model are also compared to those from a model for derailment dynamics developed using commercial software for rigid-body dynamics called Automatic Dynamic Analysis of Mechanical Systems (ADAMS). Moreover, the purpose-built and the ADAMS models produce nearly identical results, which suggest that the dynamics are being calculated correctly in both models.
\end{abstract}

\section{NOMENCLATURE}

$D_{k}=$ truck center spacing for the $k^{\text {th }}$ rail car

$E_{C}=$ lateral coupler force

$F_{C}=$ coupler force

$F_{F}=$ force at the front truck

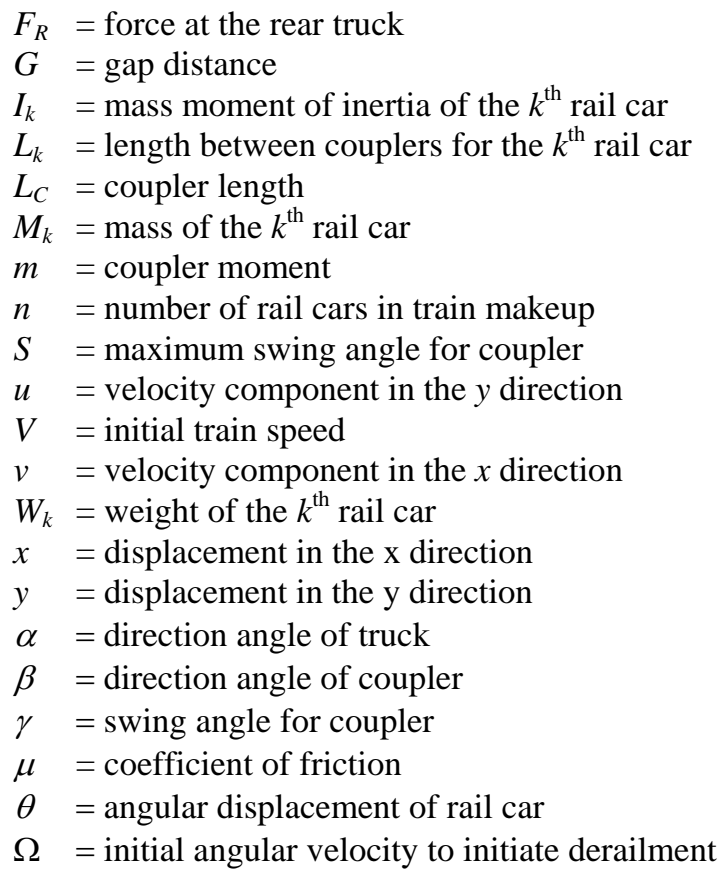

\section{INTRODUCTION}

Train derailments occur infrequently, but their consequences can be severe. Research is ongoing to develop methods to minimize and mitigate the damage resulting from train derailments. For example, accident modeling has been identified as a potential method to improve the crashworthiness of railroad tank cars [1]. Moreover, three recent accidents have focused attention on the structural integrity of railroad tank cars under impact loading conditions [2, 3, and 4].

This material is declared a work of the U.S. Government and is not subject to copyright protection in the United States. Approved for public release; distribution is unlimited. 
Sponsored by the Federal Railroad Administration (FRA), the Volpe National Transportation Systems Center is conducting and managing a research program to examine the structural integrity of railroad tank cars under accident loading conditions. The research program comprises three phases to assess the consequences of railroad tank cars involved in derailments. Each phase involves the development of computational models with different objectives: (1) dynamic modeling to estimate the gross motions of rail cars in a train derailment, (2) structural finite element modeling to calculate impact forces inferred from the gross motions, and (3) damage assessment modeling to estimate the deformations of the tank car before catastrophic failure occurs.

In this paper, the differential equations of motion are derived for a generalized train derailment scenario. These differential equations describe planar (i.e., two-dimensional) rigid-body motions of the rail cars in a right-handed and fixed (i.e., non-rotating) reference frame.

Previous work has been conducted to examine the gross motions of rail cars during a train derailment. For example, a special purpose model was developed in the 1970s [5] in which each car was assumed to behave as a rigid body with finite length but zero width. A major limitation of this model was that the cars were assumed to remain coupled during the derailment.

Another special purpose model was later developed that allowed the cars to decouple, but the criterion for decoupling was incomplete [6, 7]. A planar model was later developed at Queen's University [8], in which the differential equations of motion were derived from Lagrange's equations and were solved numerically using a special-purpose computer program written in FORTRAN.

Commercial general purpose software programs for multibody dynamics have also been used to examine the gross motions of rail cars in train derailments. For example, a threedimensional model of a 20-car train was developed using Dynamic Analysis Design Simulation (DADS) [9]. Another commercial program called Automatic Dynamic Analysis of Mechanical Systems (ADAMS) was used to develop a planar model for trains consisting up to 100 cars [10]. Results from the present work are compared directly to the ADAMS model. Explicit derivation and solution of the equations of motion can be used to provide a check on the motions calculated using the commercial programs, and vice versa.

\section{MATHEMATICAL FORMULATION}

Figure 1 shows the basic conventions for a right-handed, non-rotating description of the $k^{\text {th }}$ rail car in a train consist, where $k=1,2 \ldots n$, counting back (right to left in the figure) from the first car to derail.

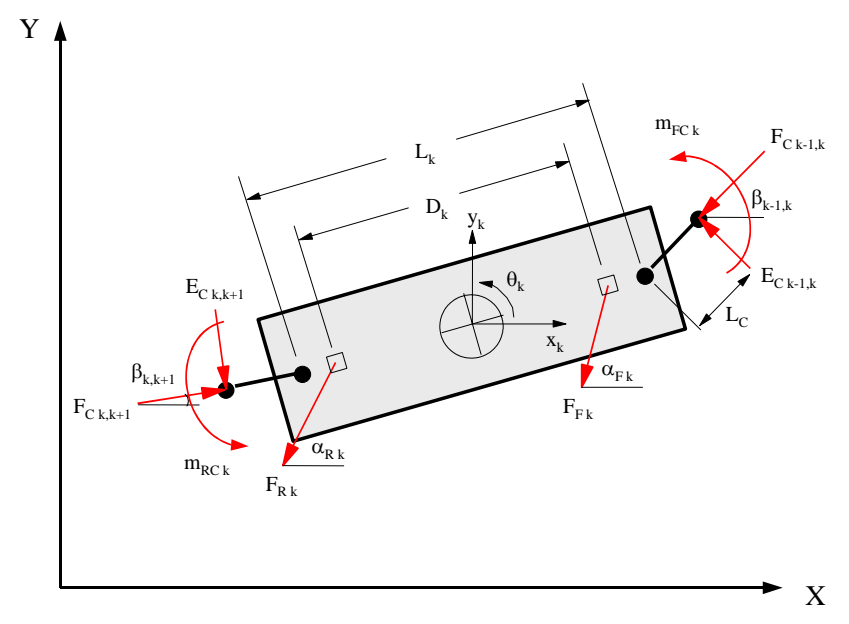

Figure 1. Rail Car Free-Body Diagram and Conventions

The gross motion for each car is characterized by the coordinates $\left(x_{k}, y_{k}, \theta_{k}\right)$ at the center of mass. The car itself is defined by its length $L_{k}$ between couplers and the distance $D_{k}$ between truck centers. The couplers are defined by a length $L_{C}$. The coordinates and the car parameters may be used to determine the positions of the front and rear couplers:

$$
\begin{array}{ll}
X_{F C, k}=x_{k}+1 / 2 L_{k} \cos \theta_{k} & y_{F C, k}=y_{k}+1 / 2 L_{k} \sin \theta_{k} \\
X_{R C, k}=x_{k}-1 / 2 L_{k} \cos \theta_{k} & y_{R C, k}=y_{k}-1 / 2 L_{k} \sin \theta_{k}
\end{array}
$$

Figure 1 also shows the definitions and positive sign conventions for the forces acting on the $k^{\text {th }}$ car. Ground forces $F_{F k}$ and $F_{R k}$ act at the front and rear trucks, respectively, at angles $\alpha_{F k}$ and $\alpha_{R k}$ with respect to the $x$-axis. The logic for determination of the ground forces and angles depends on whether the car has or has not derailed. That is, the model includes two types of friction: (1) on-track friction is an approximation of the maximum frictional force associated with emergency braking and (2) off-track friction is an estimate of the complicated resistive forces that are present when the car derails.

If the car has derailed ( $x_{k}$ equal to or greater than the $\mathrm{X}$ coordinate for the point of derailment), then $F_{F k}=F_{R k}=$ $\mu W_{k} / 2$, where $\mu$ is the ground friction coefficient and $W_{k}$ is the car's rail weight. These are assumed to be Coulomb friction forces acting opposite to the instantaneous local velocities of the respective truck centers:

This material is declared a work of the U.S. Government and is not subject to copyright protection in the United States. Approved for public release; distribution is unlimited. 
$\alpha_{F k}=\tan ^{-1}\left(v_{F k} / u_{F k}\right) \quad \alpha_{R k}=\tan ^{-1}\left(v_{R k} / u_{R k}\right)$

where

$$
\begin{array}{ll}
u_{F k}=\dot{x}_{k}-1 / 2 D_{k} \dot{\theta} \sin \theta_{k} & v_{F k}=\dot{y}_{k}+1 / 2 D_{k} \dot{\theta} \cos \theta_{k} \\
u_{R k}=\dot{x}_{k}+1 / 2 D_{k} \dot{\theta} \sin \theta_{k} & v_{R k}=\dot{y}_{k}-1 / 2 D_{k} \dot{\theta} \cos \theta_{k}
\end{array}
$$

are the truck center velocity components and the dotted quantities refer to derivatives with respect to time.

Coupler forces are positive in buff and act at angles $\beta_{k-1, k}$ and $\beta_{k, k+1}$ with respect to the $\mathrm{X}$-axis. The positions of adjacent car ends determine the angles:

$$
\begin{aligned}
& \beta_{k-1, k}=\tan ^{-1}\left[\left(y_{R C k-1}-y_{F C k}\right) /\left(x_{R C k-1}-x_{F C k}\right)\right] \\
& \beta_{k, k+1}=\tan ^{-1}\left[\left(y_{R C k}-y_{F C k+1}\right) /\left(x_{R C k}-x_{F C k+1}\right)\right]
\end{aligned}
$$

The same positions also determine the gaps between adjacent car ends:

$$
\begin{aligned}
& G_{k-1, k}=\left[\left(x_{R C k-1}-x_{F C k}\right)^{2}+\left(y_{R C k-1}-y_{F C k}\right)^{2}\right]^{1 / 2} \\
& G_{k, k+1}=\left[\left(x_{R C k}-x_{F C k+1}\right)^{2}+\left(y_{R C k}-y_{F C k+1}\right)^{2}\right]^{1 / 2}
\end{aligned}
$$

The equation of motion for the $k^{\text {th }}$ car in the $x$-direction is:

$$
\begin{aligned}
& M_{k} \ddot{x}_{k}=F_{C k, k+1} \cos \beta_{k, k+1}-F_{C k-1, k} \cos \beta_{k-1, k}-F_{R k} \cos \alpha_{R k} \\
& +E_{C k, k+1} \sin \beta_{k, k+1}-E_{C k-1, k} \cos \beta_{k-1, k}
\end{aligned}
$$

The equation of motion for the $k^{\text {th }}$ car in the $y$-direction is:

$$
\begin{aligned}
& M_{k} \ddot{y}_{k}=F_{C k, k+1} \sin \beta_{k, k+1}-F_{C k-1, k} \sin \beta_{k-1, k}-F_{R k} \sin \alpha_{R k} \\
& -E_{C k, k+1} \cos \beta_{k, k+1}+E_{C k-1, k} \cos \beta_{k-1, k}
\end{aligned}
$$

The equation of motion for the typical $k^{\text {th }}$ car in the $\theta$-direction is:

$$
\begin{aligned}
& I_{k} \ddot{\theta}_{k}=m_{R C k}+m_{\mathrm{FC} k+1} \\
& +1 / 2 L_{k}\left[F_{C k, k+1} \sin \left(\theta_{k}-\beta_{k, k+1}\right)+F_{C k-1, k} \sin \left(\theta_{k}-\beta_{k-1, k}\right)\right] \\
& +1 / 2 L_{k}\left[E_{C k, k+1} \cos \left(\theta_{k}-\beta_{k, k+1}\right)+E_{C k-1, k} \cos \left(\theta_{k}-\beta_{k-1, k}\right)\right] \\
& +1 / 2 D_{k}\left[F_{F k, k+1} \sin \left(\theta_{k}-\alpha_{F k}\right)+F_{F k} \sin \left(\theta_{k}-\alpha_{R k}\right)\right]
\end{aligned}
$$

The coupler hardware between two cars comprises two sets of knuckle, shank, and draft gear. For the purposes of the present model, the two sets are treated as a single pin-ended bar, with the coupler force $F_{C}$ aligned along the bar, provided that neither individual swing angle limit has been reached. The bar may begin to support a moment at one end, when an angle limit is reached, and may support moments at both ends if both angle limits have been reached. Since the bar is also assumed to have no mass, force and moment equilibrium conditions applied to it require the presence of lateral forces, as well as the aligned force $F_{C}$. Figure 2 shows the coupler free-body diagram, together with the reactions on the cars, for the typical coupler between car $k$ and car $k+1$.

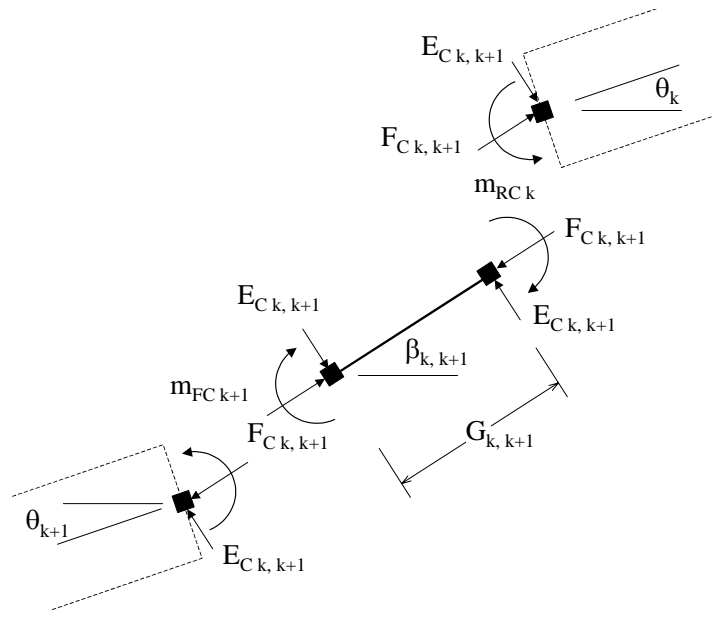

Figure 2. Coupler Free-Body Diagram

Figure 3 illustrates the coupler force curve assumed in the model. This characteristic includes a dead band or gap $(0.25$ feet assumed) to simulate slack and free translation, which is centered about a nominal coupler length of 4 feet. The coupler stiffness is assumed to be $50 \times 10^{6} \mathrm{lb} / \mathrm{ft}$.

This material is declared a work of the U.S. Government and is not subject to copyright protection in the United States. Approved for public release; distribution is unlimited. 


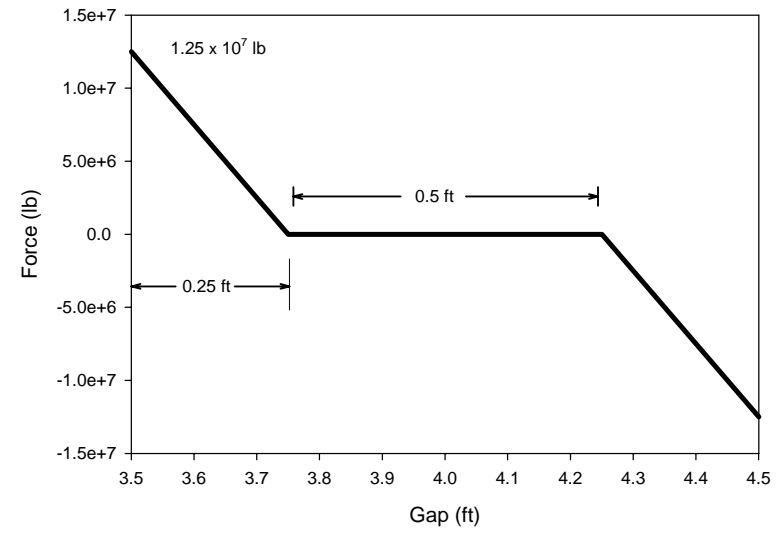

Figure 3. Assumed Coupler Force Characteristic

In addition to the aligned coupler forces $E_{C k, k+1}$, Figure 1 shows lateral forces $E_{C k, k+1}$, a coupler moment $m_{R C k}$ at the rear end of car $k$, and a coupler moment $m_{F C k+1}$ at the front end of car $k+1$. The positive sign conventions for the moments are defined such that they are in the directions shown when $\beta_{k, k+1}$ exceeds $\theta_{k}$ and $\theta_{k+1}$. For the front and rear couplers on the typical car $k$, the swing angles are:

$$
\gamma_{F C k}=\beta_{k-1, k}-\theta_{k} \quad \gamma_{R C k}=\beta_{k, k+1}-\theta_{k}
$$

The moments are computed from these swing angles.

The lateral force is derived from the moment equilibrium condition for the coupler:

$E_{C k, k+1}=\left(m_{R C k}+m_{F C k+1}\right) / G_{k, k+1}$

where $G_{k, k+1}$ is the current value of the coupler gap.

Figure 4 shows the coupler moment characteristic assumed in the model. It includes a dead space between swings of $\pm 17.5^{\circ}$ with a sudden rise when the swing angle is greater than the maximum swing. In practice, the maximum swing angle and moment would depend on the type of coupler (e.g., plainE, shelf-E, etc.).

\section{Initial conditions}

The train consist is initialized in a slack configuration. The point of derailment in the model is assumed to be the center of mass for the lead car. Therefore, with the first car center of mass at $x_{1}$ equal to zero (i.e., point of derailment), the trailing car centers are set equal to

$x_{k}=-\sum_{j=1}^{k-1} x_{j}+\frac{1}{2}\left(L_{j}+L_{j+1}\right)+G_{0} \quad$ for $k=2,3 \cdots n$ where $G_{o}$ is equal to 4 feet. This equation describes the midslack condition, which is an arbitrary choice, but avoids the introduction of large initial coupler forces and has some degree of relation to real situations. The initial conditions for all car center coordinates $\left(y_{k}, \theta_{k}\right)$ are zero. All initial gaps are $G_{k, k+1}=G_{0}$. All other initial angles $\alpha_{F k}, \alpha_{R k}$, and $\beta_{k, k+1}$ are zero.

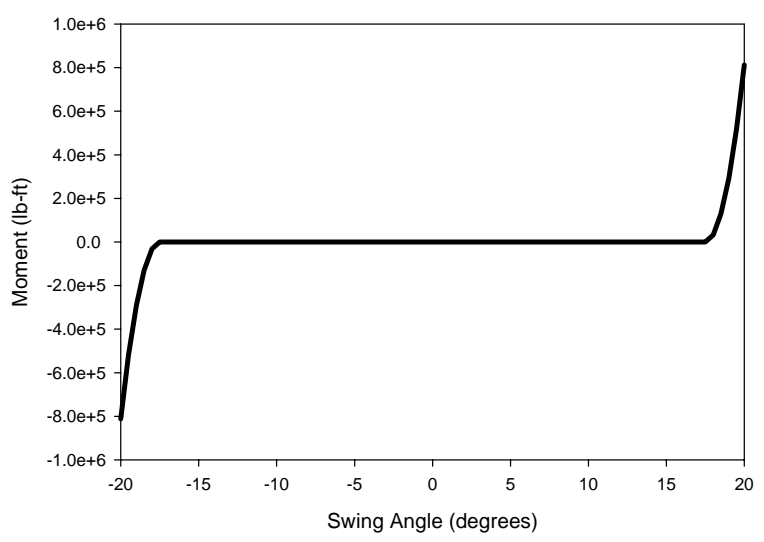

Figure 4. Assumed Coupler Moment Characteristic

\section{Initial conditions for rate variables}

In general, the motion of the train in the generalized derailment presented here begins with an initial rotation imparted to the lead car and with all cars traveling at a given initial speed. Thus, the initial velocities are

$\dot{x}_{k}=V \quad \dot{y}_{k}=0$

for all cars where $V$ is the initial train speed. The initial angular velocities are

$$
\dot{\theta}_{1}=\Omega \text { and } \dot{\theta}_{k}=0 \text { for } k=2,3 \cdots n
$$

where $\Omega$ is the initial rotational speed of the first car to start the pileup.

With these initial conditions, the lead car swings out in the direction of the initial rotation, with higher off-track frictional forces applied at the trucks opposing the direction of motion. As the cars derail, the coupler forces between cars create a moment couple. The many highly coupled interactions cause the rail cars to form irregular buckling patterns.

Numerical solution to the equations of motion is carried out using a Runge-Kutta algorithm with an adaptive time step [11], which is coded into a purpose-built FORTRAN computer program.

This material is declared a work of the U.S. Government and is not subject to copyright protection in the United States. Approved for public release; distribution is unlimited. 


\section{SENSITIVITY STUDIES AND RESULTS}

Sensitivity studies are conducted using the purpose-built model for derailment dynamics. Baseline values were assumed and varied one factor at a time to examine the relative effect of various factors on the derailment outcomes in terms of (1) the number of derailed cars, (2) the maximum closing speeds, and (3) peak coupler forces. Closing speed or velocity is the relative difference in speed or velocity between two impacting cars. Table 1 lists the baseline values assumed in the sensitivity studies.

Table 1. Baseline Values for Sensitivity Studies

\begin{tabular}{|l|l|}
\hline Parameter & Baseline Value \\
\hline Train length & 60 cars \\
\hline Car weight & $150,000 \mathrm{lb}$ \\
\hline Initial translational velocity & $\begin{array}{l}37 \text { miles per hour } \\
\text { (mph) }\end{array}$ \\
\hline Initial angular velocity & 0.2 radian per second \\
\hline On-track coefficient of friction & 0.15 \\
\hline Off-track coefficient of friction & 0.50 \\
\hline
\end{tabular}

Each parameter was varied from its baseline value by \pm 20 percent and \pm 50 percent while the remaining inputs, including car length and coupler characteristics, were set equal to their respective baseline values. Table 2 lists the range of values assumed for each factor in the sensitivity studies.

Table 2. Range of Values for Sensitivity Studies

\begin{tabular}{|l|l|}
\hline Parameter & Range \\
\hline Train length & 30 to 90 cars \\
\hline Car weight & 75 to 225 kips \\
\hline Initial translational velocity & 18.5 to $55.5 \mathrm{mph}$ \\
\hline Initial angular velocity & 0.1 to 0.3 radian per sec \\
\hline On-track coefficient of friction & 0.075 to 0.225 \\
\hline Off-track coefficient of friction & 0.25 to 0.75 \\
\hline
\end{tabular}

Figure 5 shows the relative effect of changing each parameter on the number of derailed cars calculated by the purpose-built model. The baseline case results in 13 cars derailing from the 60-car train. Clearly, train speed has the greatest effect on the number of derailed cars. Increasing the train speed by 50 percent over the baseline increases the number of derailed cars from 13 to 24 . Decreasing the initial train speed to 50 percent below the baseline reduces the number of derailed cars from 13 to 6 . Friction also has a significant effect on the number of derailed cars. The relative difference between ground and rail friction coefficients is proportional to the force to decelerate the train. Greater differences in friction coefficients lead to greater retarding forces which slow the train. Moreover, friction forces have an inverse relationship compared to the other inputs. That is, increasing friction reduces the derailment severity in terms of the number of derailed cars while increasing the value of the other parameters increases the number of derailed cars.

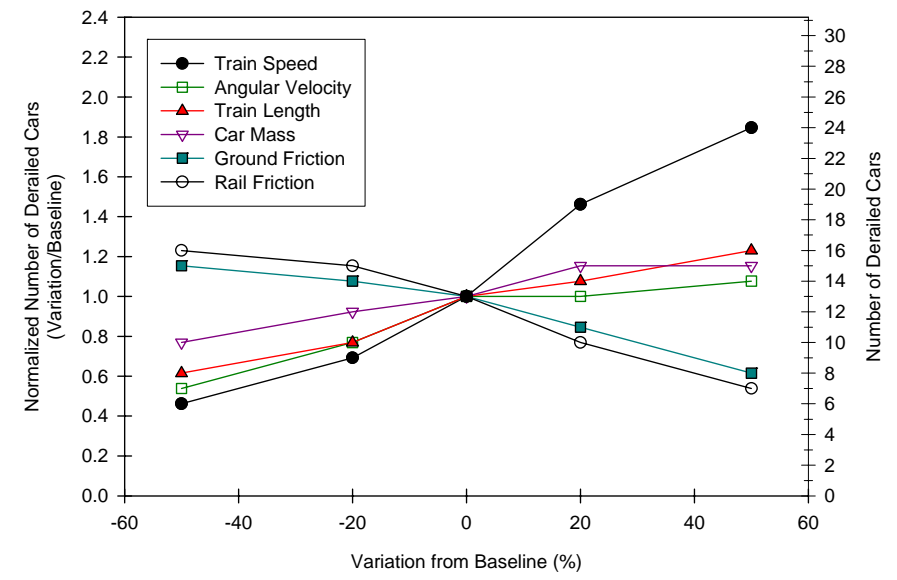

Figure 5. Effect of Factors on Number of Derailed Cars

Figure 6 shows the relative effect of changing each parameter on the maximum closing velocity calculated using the purpose-built model. The baseline case results in a maximum closing velocity of $19 \mathrm{mph}$. Train speed has the most significant effect on maximum closing velocity. Increasing the initial train speed by 50 percent over the baseline increases the maximum closing velocity by almost 60 percent. Decreasing the initial train speed by 50 percent below the baseline reduces the maximum closing velocity by 30 percent. Ground friction has a somewhat moderate effect on closing velocity. Increasing ground friction by 50 percent over the baseline increases maximum closing velocity by more than 20 percent. Changing the other input parameters varies the maximum closing velocity by at most \pm 15 percent.

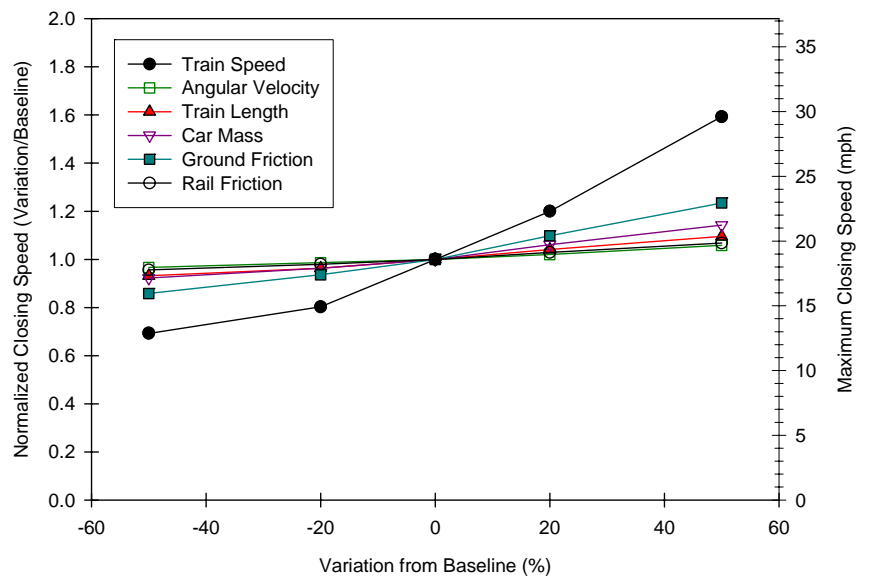

This material is declared a work of the U.S. Government and is not subject to copyright protection in the United States. Approved for public release; distribution is unlimited. 


\section{Figure 6. Effect of Factors on Maximum Closing Velocity}

The relatively weak effect of initial rotational velocity on closing velocity means that the cause of the derailment (e.g., broken rail, wheel climb, excessive lateral-to-vertical wheel load ratio, etc.) does not have a significant effect on the maximum closing velocities in car-to-car impacts.

If the coupler forces are relatively large, couplers can break and may potentially become impacting objects. Figure 7 shows the relative effect of changing each parameter on the peak coupler force calculated by the purpose-built model. The peak coupler force corresponding to the baseline case is 1,821 kips. Increasing the initial train speed by 50 percent over the baseline value increases the peak coupler force by almost twice.

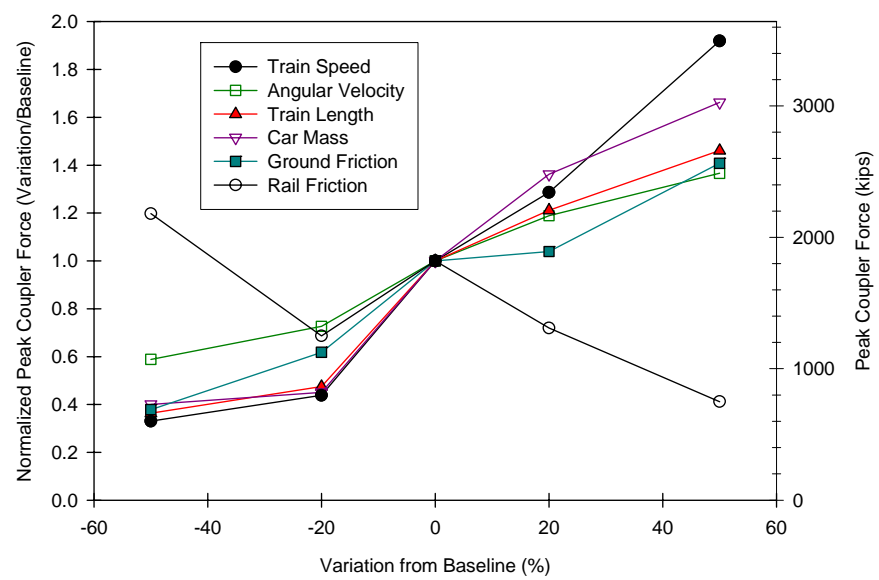

Figure 7. Effect of Factors on Peak Coupler Force

Repeating the sensitivity studies using the ADAMS model for derailment dynamics gives nearly identical results, which are presented in the appendix.

\section{CONCLUDING REMARKS}

This paper describes a purpose-built model to examine the dynamics of rail cars in a generalized train derailment. The equations of motion were derived explicitly from Newton's laws of physics, and were coded into a built-for-purpose FORTRAN computer program. Sensitivity analyses were performed with the model to examine the relative effect of various factors on derailment severity in terms of the number of derailed cars, maximum closing speeds for colliding cars, and peak coupler forces that may potentially become impacting forces.

The results from the purpose-built model were compared to those calculated using a commercial software code called ADAMS [10]. The computational times to run the purposebuilt FORTRAN model were much less than the ADAMS model (minutes versus hours). Moreover, the two models produced virtually identical results. This comparison provided verification of both analyses.

The similarity of results from the purpose-built model presented in this paper and the ADAMS model suggests that the dynamics are being calculated correctly for the assumed scenarios in both models. However, the assumptions at best provide only a simplified representation of actual conditions. For example, it is unlikely that the actual terrain at a derailment site will be perfectly level. In addition, the effective friction between derailed cars and the ground is likely to vary. Thus, the models provide a general representation but do not reproduce specific derailment events.

Nevertheless, the general results can provide some useful insights. For example, the closing velocities in post-derailment car-to-car impacts appear to average about half of the initial train speed.

Another general result is that train speed appears to be the most important variable. The assumed train speed was increased by a factor of 3 (18.5 to $55.5 \mathrm{mph}$ ) in a series of cases, with the following outcomes: (1) the number of derailed cars increased by a factor of 4, (2) closing speed for potential collisions increased by a factor of 2.5 , and (3) peak coupler force increased by a factor of 6 .

\section{ACKNOWLEDGMENTS}

The Federal Railroad Administration (FRA), Office of Research and Development sponsored the work described in this paper. Ms. Claire Orth is the Chief of the Equipment and Operating Practices Division. Mr. Francisco Gonzalez is the project manager for research on railroad tank cars. Mr. Eloy Martinez also provides technical direction to this project.

\section{REFERENCES}

[1] Transportation Research Board, 1994: “Ensuring Tank Car Safety,” TRB Special Report 243, Washington, DC.

[2] National Transportation Safety Board, 2004: “Derailment of Canadian Pacific Railway Freight Train 292-16 and Subsequent Release of Anhydrous Ammonia Near Minot, North Dakota January 18, 2002,” Railroad Accident Report NTSB/RAR-04/01.

[3] National Transportation Safety Board, 2006: “Collision of Union Pacific Railroad Train MHOTU-23 with BNSF Railway Company Train MEAP-TUL-126-D With Subsequent Derailment and Hazardous Materials Release, Macdona, Texas, June 28, 2004,” Railroad Accident Report NTSB/RAR-06/03.

[4] National Transportation Safety Board, 2005: “Collision of Norfolk Southern Freight Train 192 with Standing Norfolk Southern Local Train P22 With Subsequent Hazardous Materials Release at Graniteville, South Carolina January 6, 2005,” Railroad Accident Report NTSB/RAR-05/04.

This material is declared a work of the U.S. Government and is not subject to copyright protection in the United States. Approved for public release; distribution is unlimited. 
[5] Yang, T.H., Manos, W.B., Johnstone, B., 1972: “Dynamic Analysis of Train Derailments," ASME Winter Annual Meeting, ASME Paper 72-WA/RT-5.

[6] Birk, A.M., Anderson, R.J., Coppens, A.J., 1990: "Computer simulation of a derailment accident, Part I Model basis,” Journal of Hazardous Materials 25, 121147.

[7] Birk, A.M., Anderson, R.J., Coppens, A.J., 1990: "Computer simulation of a derailment accident, Part II Sample simulation,” Journal of Hazardous Materials 25, 149-165.

[8] Toma, E.E., 1998: "A Computer Model of a Train Derailment,” Ph.D. Thesis, Queen's University, Kingston, Ontario, Canada.

[9] Han, H.S., Koo, J.S., 2003: "Simulation of train crash in three dimensions," Vehicle System Dynamics 20, 435-450.

[10] Paetsch, C.R., Perlman, A.B., Jeong, D.Y., 2006: "Dynamic Simulation of Train Derailments," Proceedings of IMECE2006, 2006 ASME International Mechanical Engineering Congress and Exposition, IMECE200614607.

[11]Press, W.H., Teukolsky, S.A., Vetterling, W.T., Flannery, B.P., 1992: Numerical Recipes in FORTRAN: The Art of Scientific Computing, $2^{\text {nd }}$ edition, Cambridge University Press, New York, NY.

\section{APPENDIX}

Figure 8 shows the relative effect of the different factors on the number of derailed cars. The purpose-built and ADAMS models calculate 13 derailed cars for the baseline case. The ADAMS model calculates slightly more derailed cars as the train speed increases than the purpose-built model (see Figure 5). The ADAMS results also show a slightly greater effect of friction on the number of derailed cars than the purpose-built model, which is attributed to different numerical methods used in the two models to solve the equations of motion.

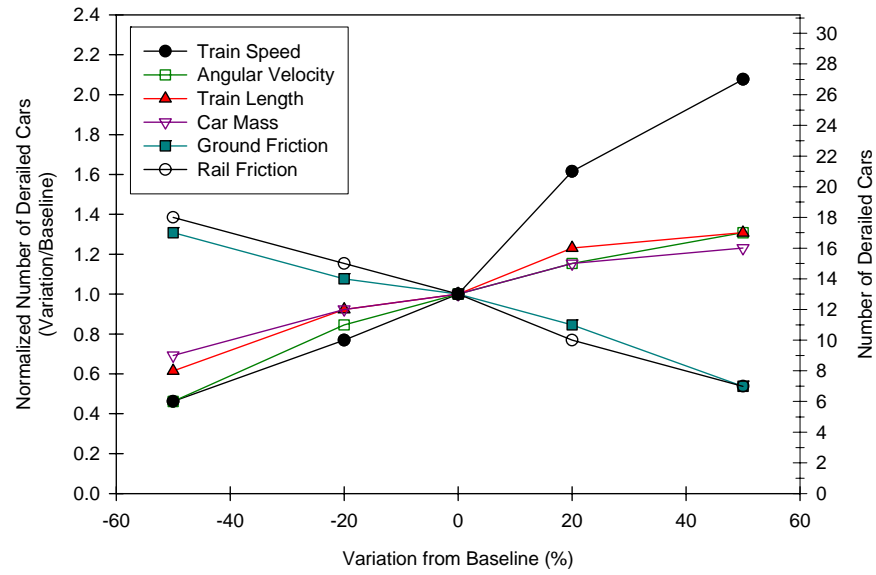

\section{Figure 8. Effect of Factors on Number of Derailed Cars} using ADAMS Model

Figure 9 shows the variations in maximum closing speeds calculated using the ADAMS model, which are similar to those from the purpose-built model (see Figure 6). Both models calculate a maximum closing speed of $19 \mathrm{mph}$ for the baseline case.

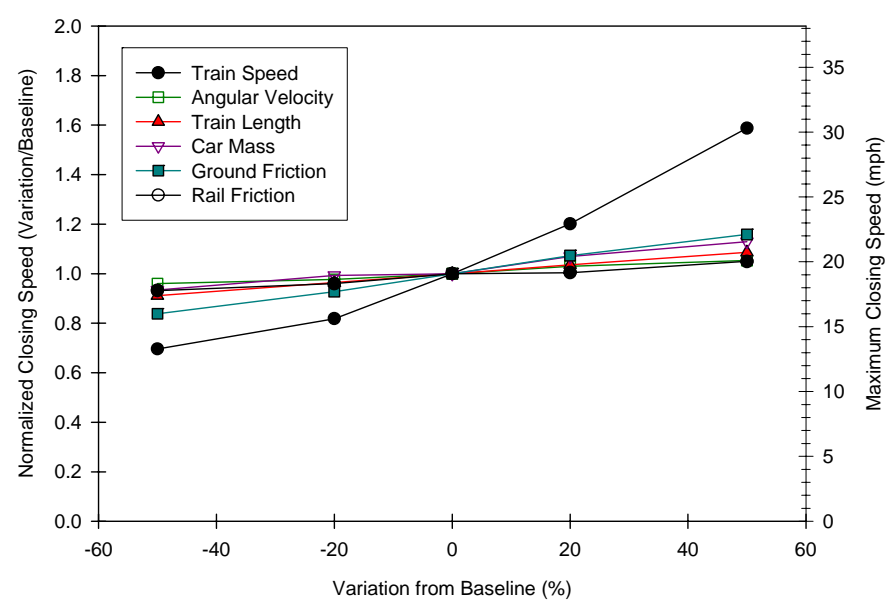

Figure 9. Effect of Factors on Maximum Closing Velocity using ADAMS Model

Figure 10 shows the relative effect of changing each factor on the peak coupler force calculated using the ADAMS model. For the baseline case, the ADAMS model calculates a peak coupler force of 1,858 kips (compared to 1,821 kips from the purpose-built model). Overall, the variations in peak coupler forces calculated by both models are similar (see Figure 7).

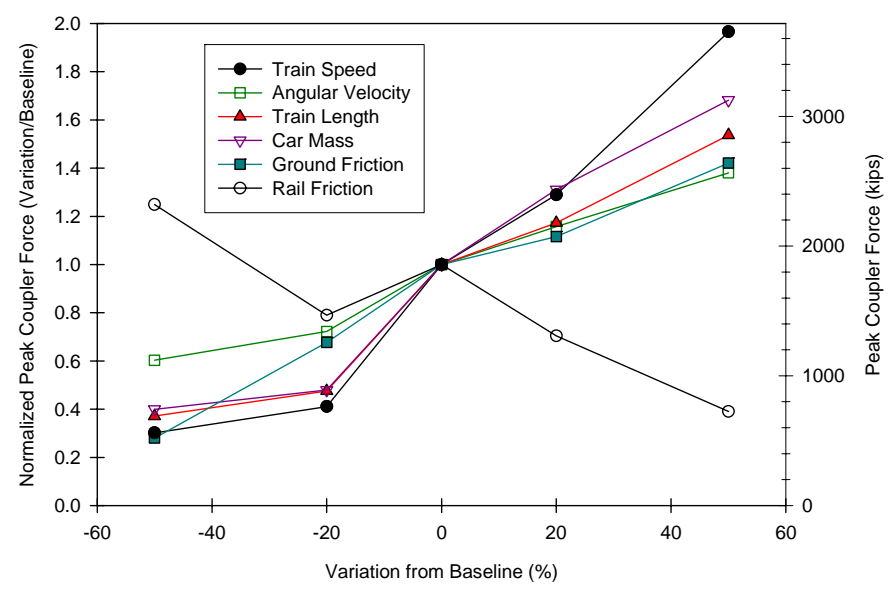

Figure 10. Effect of Factors on Peak Coupler Force using ADAMS Model

This material is declared a work of the U.S. Government and is not subject to copyright protection in the United States. Approved for public release; distribution is unlimited. 
This material is declared a work of the U.S. Government and is not subject to copyright protection in the United States. Approved for public release; distribution is unlimited. 